Supplement of

\title{
Structural changes to forests during regeneration affect water flux partitioning, water ages and hydrological connectivity: Insights from tracer-aided ecohydrological modelling
}

Aaron J. Neill et al.

Correspondence to: Aaron J. Neill (aaron.neill@abdn.ac.uk)

The copyright of individual parts of the supplement might differ from the article licence. 
Table S1: Forcing data used for $\mathrm{EcH}_{2} \mathrm{O}$-iso

5

\begin{tabular}{llll} 
Forcing dataset(s) & Source & Temporal coverage & Notes \\
\hline Precipitation & $\begin{array}{l}\text { Interpolated using an inverse distance- } \\
\text { weighted algorithm and five nearby }(<10\end{array}$ & Prior to July 2014 & c.f. Capell et al. (2012) \\
& $\mathrm{km})$ gauges
\end{tabular}

Up to three automated weather stations in the

catchment July 2014 onwards

\begin{tabular}{|c|c|c|c|}
\hline Minimum and maximum & ERA-interim climate reanalysis & Prior to July 2014 & See Dee et al. (2011) \\
\hline & $\begin{array}{l}\text { Up to three automated weather stations in the } \\
\text { catchment }\end{array}$ & July 2014 onwards & - \\
\hline \multirow{2}{*}{$\begin{array}{l}\text { Mean air temperature, } \\
\text { relative humidity and } \\
\text { windspeed }\end{array}$} & Balmoral weather station $\sim 5 \mathrm{~km}$ away & Prior to July 2014 & See Met Office (2017) \\
\hline & $\begin{array}{l}\text { Up to three automated weather stations in the } \\
\text { catchment }\end{array}$ & July 2014 onwards & - \\
\hline $\begin{array}{l}\text { Short- and long-wave } \\
\text { radiation }\end{array}$ & ERA-interim climate reanalysis & Full simulation period & See Dee et al. (2011) \\
\hline $\begin{array}{l}\text { Isotopic }\left(\delta^{2} \mathrm{H}\right) \\
\text { composition of } \\
\text { precipitation }\end{array}$ & ISCO 3700 sampler at catchment outlet & Full simulation period & $\begin{array}{l}\text { Daily bulk samples were } \\
\text { collected and preserved } \\
\text { under a layer of paraffin. A } \\
\text { Los Gatos DLT-100 was } \\
\text { used for isotope analysis } \\
\left(\delta^{2} \mathrm{H} \text { precision: } \pm 0.4 \%\right) \text {. }\end{array}$ \\
\hline
\end{tabular}


Table S2: The sampling ranges and $90 \%$-spread calibrated ranges of soil, vegetation and channel parameters identified as sensitive in this application of EcH2O-iso. Additional information on parameter definitions can be found at: https://ech2oiso.readthedocs.io/en/latest/Setup.html

\begin{tabular}{|c|c|c|c|c|}
\hline \multirow{2}{*}{$\begin{array}{l}\text { Parameter } \\
\text { Soil }\end{array}$} & \multicolumn{4}{|c|}{ Sampling range $[90 \%$ spread calibrated range] } \\
\hline & Peat & Peaty gley & Podzol & Ranker \\
\hline \multirow[t]{2}{*}{ Air entry pressure $(\mathrm{m})$} & $0.01-0.45$ & $0.01-0.1$ & $0.01-0.1$ & $0.05-0.2$ \\
\hline & {$[0.05-0.41]$} & {$[0.03-0.09]$} & {$[0.02-0.09]$} & {$[0.06-0.17]$} \\
\hline \multirow{2}{*}{ Brooks-Corey lambda (-) } & $3.0-8.0$ & $3.0-8.0$ & $3.0-8.0$ & $3.0-8.0$ \\
\hline & {$[3.1-7.8]$} & {$[4.9-7.8]$} & {$[4.1-7.0]$} & {$[3.4-7.4]$} \\
\hline \multirow[t]{2}{*}{ Soil L1 depth (m) } & $0.05-0.15$ & $0.05-0.15$ & $0.05-0.15$ & $0.05-0.15$ \\
\hline & {$[0.05-0.13]$} & {$[0.05-0.14]$} & {$[0.06-0.13]$} & {$[0.06-0.14]$} \\
\hline \multirow[t]{2}{*}{ Soil L2 depth (m) } & $0.05-0.20$ & $0.05-0.20$ & $0.05-0.20$ & $0.05-0.20$ \\
\hline & {$[0.05-0.19]$} & {$[0.07-0.18]$} & {$[0.06-0.20]$} & {$[0.06-0.19]$} \\
\hline \multirow[t]{2}{*}{ Total soil depth (m) } & $0.5-40.0$ & $0.5-40.0$ & $0.5-10.0$ & $0.5-5.0$ \\
\hline & {$[0.7-36.4]$} & [1.1-27.2] & {$[1.7-8.3]$} & {$[0.7-4.6]$} \\
\hline \multirow{2}{*}{$\begin{array}{l}\text { Saturated horizontal hydraulic } \\
\text { conductivity }\left(\mathrm{ms}^{-1}\right)\end{array}$} & $1.0 \times 10^{-5}-1 \times 10^{-2}$ & $1.0 \times 10^{-5}-1.0 \times 10^{-3}$ & $1.0 \times 10^{-5}-1.0 \times 10^{-3}$ & $1.0 \times 10^{-6}-1.0 \times 10^{-4}$ \\
\hline & {$\left[1.6 \times 10^{-5}-3.6 \times 10^{-3}\right]$} & {$\left[2.2 \times 10^{-5}-8.5 \times 10^{-4}\right]$} & {$\left[4.6 \times 10^{-4}-9.1 \times 10^{-4}\right]$} & {$\left[1.2 \times 10^{-6}-8.2 \times 10^{-5}\right]$} \\
\hline \multirow[t]{2}{*}{ Anisotropy (-) } & $1.0 \times 10^{-3}-1.0$ & $1.0 \times 10^{-3}-0.6$ & $1.0 \times 10^{-3}-0.6$ & $1.0 \times 10^{-3}-0.6$ \\
\hline & {$\left[1.6 \times 10^{-3}-0.85\right]$} & {$\left[1.8 \times 10^{-3}-0.35\right]$} & {$\left[1.3 \times 10^{-3}-0.53\right]$} & {$\left[1.1 \times 10^{-3}-0.22\right]$} \\
\hline \multirow{2}{*}{$\begin{array}{l}\text { Conductivity exponential decay constant } \\
\left(\mathrm{m}^{-1}\right)\end{array}$} & $1.0-5.0$ & $1.0-5.0$ & $1.0-5.0$ & $1.0-5.0$ \\
\hline & {$[1.2-4.8]$} & [1.9-4.6] & {$[1.5-4.9]$} & {$[1.3-4.7]$} \\
\hline \multirow[t]{2}{*}{ Porosity $\left(\mathrm{m}^{3} \mathrm{~m}^{-3}\right)$} & $0.8-0.98$ & $0.7-0.9$ & $0.4-0.7$ & $0.4-0.6$ \\
\hline & {$[0.81-0.93]$} & {$[0.74-0.89]$} & {$[0.50-0.69]$} & {$[0.42-0.57]$} \\
\hline \multirow[t]{2}{*}{ Porosity exponential decay constant $\left(\mathrm{m}^{-1}\right)$} & $5.0-10.0$ & $5.0-10.0$ & $3.0-5.0$ & $0.5-1.0$ \\
\hline & {$[5.4-9.7]$} & {$[5.5-9.3]$} & [3.2-4.9] & {$[0.6-1.0]$} \\
\hline Vegetation & Pre-existing pine & Heather & Sphagnum & Molinia grass \\
\hline \multirow[t]{2}{*}{$\operatorname{LAI}\left(\mathrm{m}^{2} \mathrm{~m}^{-2}\right)$} & $2.0-4.0$ & $1.4-2.0$ & $2.0-3.5$ & $1.0-3.0$ \\
\hline & {$[2.2-3.8]$} & {$[1.5-2.0]$} & {$[2.1-3.4]$} & {$[1.2-2.8]$} \\
\hline \multirow[t]{2}{*}{ Maximum canopy water storage $\left(\mathrm{m} \mathrm{LAI}^{-1}\right)$} & $3.0 \times 10^{-4}-3.0 \times 10^{-3}$ & $5.0 \times 10^{-4}-2.0 \times 10^{-3}$ & $1.0 \times 10^{-3}-1.0 \times 10^{-2}$ & $1.0 \times 10^{-4}-5.0 \times 10^{-4}$ \\
\hline & {$\left[3.5 \times 10^{-4}-2.7 \times 10^{-3}\right]$} & {$\left[5.4 \times 10^{-4}-1.7 \times 10^{-3}\right]$} & {$\left[1.2 \times 10^{-3}-7.9 \times 10^{-3}\right]$} & {$\left[1.4 \times 10^{-4}-4.7 \times 10^{-4}\right]$} \\
\hline \multirow[t]{2}{*}{ Maximum stomatal conductance $\left(\mathrm{ms}^{-1}\right)$} & $3.3 \times 10^{-3}-8.1 \times 10^{-3}$ & $5.2 \times 10^{-3}-6.6 \times 10^{-3}$ & $1.3 \times 10^{-2}-1.8 \times 10^{-2}$ & $6.4 \times 10^{-3}-1.5 \times 10^{-2}$ \\
\hline & {$\left[3.6 \times 10^{-3}-7.9 \times 10^{-3}\right]$} & {$\left[5.3 \times 10^{-3}-6.5 \times 10^{-3}\right]$} & {$\left[1.3 \times 10^{-2}-1.8 \times 10^{-2}\right]$} & {$\left[6.6 \times 10^{-3}-1.4 \times 10^{-2}\right]$} \\
\hline \multirow[t]{2}{*}{ Stomatal sensitivity to light (-) } & $200-500$ & $200-500$ & $200-500$ & $200-500$ \\
\hline & [213-452] & [223-442] & [220-496] & [222-479] \\
\hline \multirow{2}{*}{$\begin{array}{l}\text { Stomatal sensitivity to vapour pressure } \\
\text { deficit (-) }\end{array}$} & $1.0 \times 10^{-3}-3.0 \times 10^{-3}$ & $1.0 \times 10^{-3}-3.0 \times 10^{-3}$ & $1.0 \times 10^{-3}-3.0 \times 10^{-3}$ & $1.0 \times 10^{-3}-3.0 \times 10^{-3}$ \\
\hline & {$\left[1.9 \times 10^{-3}-2.9 \times 10^{-3}\right]$} & {$\left[1.1 \times 10^{-3}-2.9 \times 10^{-3}\right]$} & {$\left[1.1 \times 10^{-3}-2.9 \times 10^{-3}\right]$} & {$\left[1.1 \times 10^{-3}-2.8 \times 10^{-3}\right]$} \\
\hline \multicolumn{5}{|l|}{ Soil water potential (-MPa): } \\
\hline \multirow{2}{*}{$\begin{array}{l}\text { - Causing complete stomatal } \\
\text { closure }\end{array}$} & $1.5-6.0$ & $1.5-6.0$ & $1.5-6.0$ & $1.5-6.0$ \\
\hline & {$[1.8-5.8]$} & {$[1.8-5.7]$} & {$[1.8-5.6]$} & {$[1.6-5.8]$} \\
\hline $\begin{array}{l}\text { - No longer limiting stomatal } \\
\text { conductance }\end{array}$ & $0.1-1.0$ & $0.1-1.0$ & $0.1-1.0$ & $0.1-1.0$ \\
\hline
\end{tabular}




\begin{tabular}{|c|c|c|c|c|}
\hline & {$[0.14-0.95]$} & {$[0.20-0.96]$} & {$[0.22-0.89]$} & {$[0.14-0.92]$} \\
\hline \multirow[t]{2}{*}{ Minimum temperature of comfort $\left({ }^{\circ} \mathrm{C}\right)$} & $-5.0--3.0$ & $-5.0--3.0$ & $-5.0--3.0$ & $-6.0--3.0$ \\
\hline & {$[-5.0--3.1]$} & {$[-4.8--3.2]$} & {$[-4.8--3.3]$} & {$[-5.8--3.1]$} \\
\hline \multirow[t]{2}{*}{ Optimal temperature $\left({ }^{\circ} \mathrm{C}\right)$} & $10.0-25.0$ & $15.0-25.0$ & $10.0-18.0$ & $12.0-18.0$ \\
\hline & {$[11.0-24.2]$} & {$[15.3-24.4]$} & {$[10.3-17.3]$} & {$[12.2-17.2]$} \\
\hline \multirow[t]{2}{*}{ Maximum temperature of comfort $\left({ }^{\circ} \mathrm{C}\right)$} & $35.0-42.0$ & $40.0-45.0$ & $38.0-42.0$ & $30.0-40.0$ \\
\hline & {$[35.4-41.4]$} & {$[40.5-44.8]$} & [38.1-41.7] & {$[31.7-39.1]$} \\
\hline \multirow[t]{2}{*}{ Light attenuation coefficient (-) } & $0.3-0.6$ & $0.3-0.6$ & $0.3-0.6$ & $0.3-0.6$ \\
\hline & {$[0.33-0.58]$} & {$[0.36-0.58]$} & {$[0.39-0.60]$} & {$[0.33-0.58]$} \\
\hline Vertical root distribution exponential & $10.0-20.0$ & $27.0-40.0$ & $27.0-100.0$ & $6.0-10.0$ \\
\hline decay constant $\left(\mathrm{m}^{-1}\right)$ & {$[10.9-19.5]$} & {$[28.1-39.2]$} & {$[32.5-82.8]$} & {$[6.6-10.0]$} \\
\hline \multicolumn{5}{|l|}{ Channel } \\
\hline \multirow{2}{*}{$\begin{array}{l}\text { Channel resistance to groundwater } \\
\text { seepage (-) }\end{array}$} & $0.01-0.05$ & & & \\
\hline & {$[0.01-0.04]$} & & & \\
\hline \multirow[t]{2}{*}{ Manning's n } & $1.0-50.0$ & & & \\
\hline & {$[4.8-49.1]$} & & & \\
\hline
\end{tabular}

10 


\section{Supplementary figures}

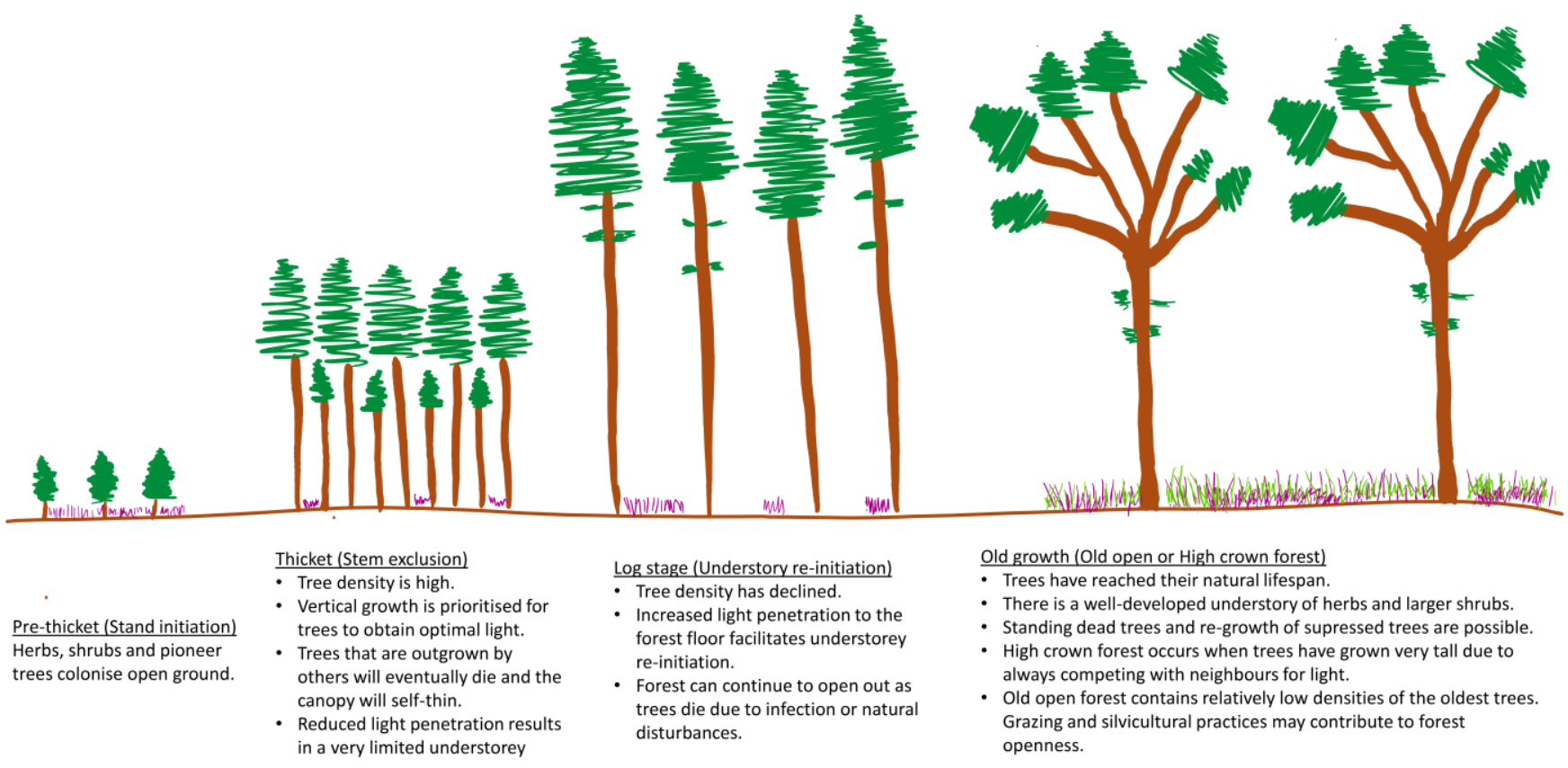

Figure S1: Summary of natural pinewood regeneration. After Summers (2018) and Summers et al. (2008). 

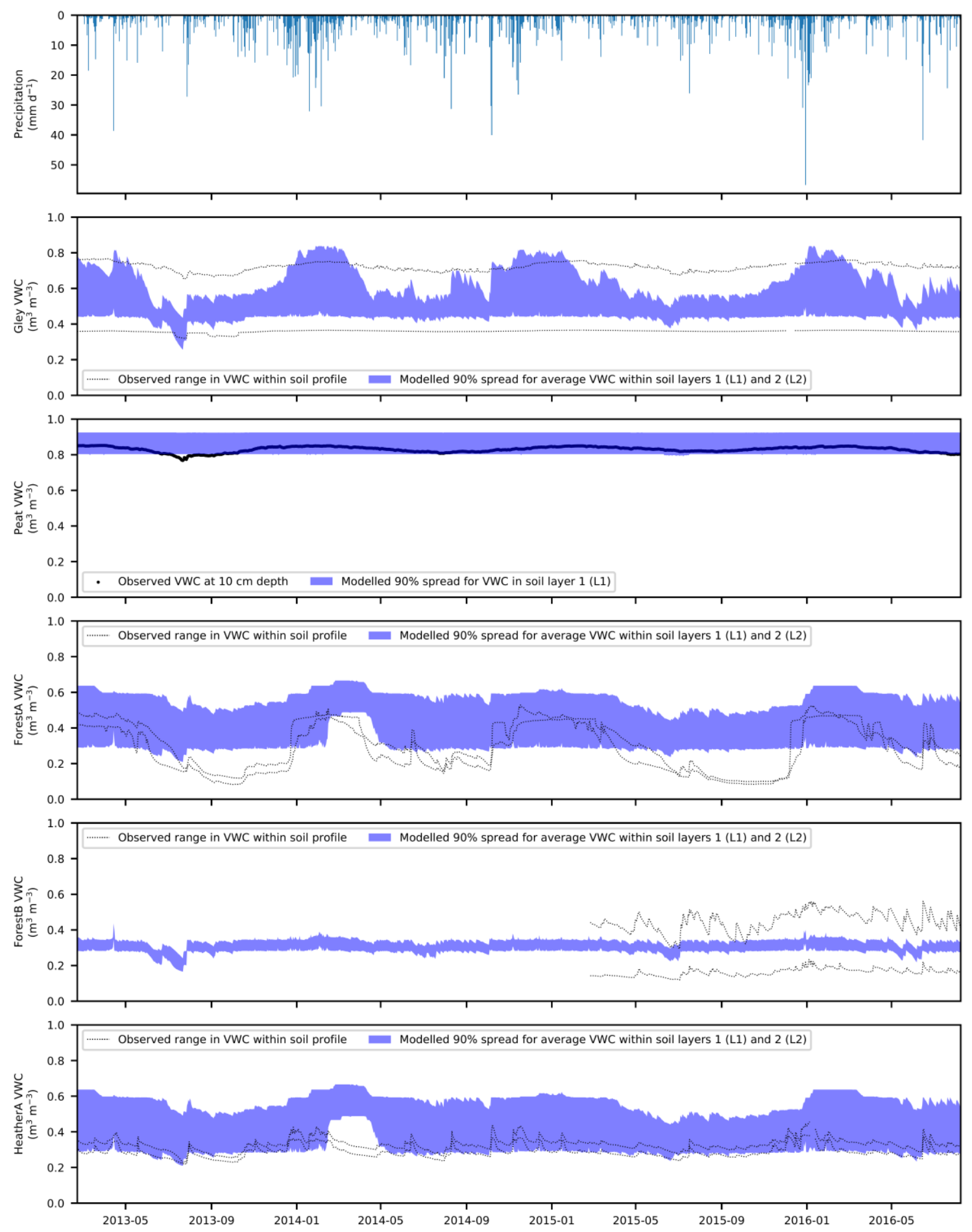

Figure S2: Time series of observed and simulated volumetric water content (VWC) at sites not shown in Figure 3.90\% spread of simulations are from the 30 behavioural model runs. 

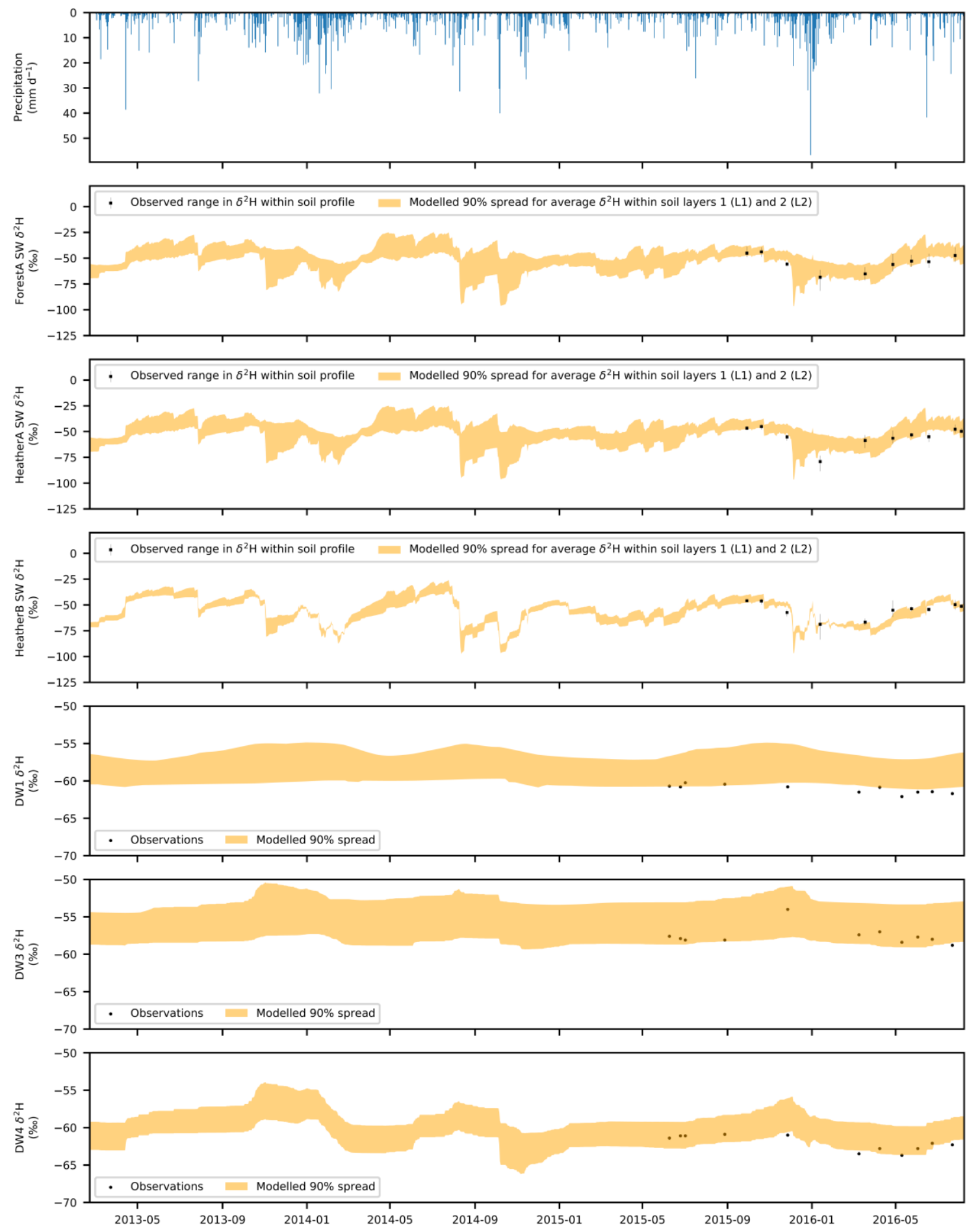

Figure S3: Time series of observed and simulated soil water (SW) and deeper groundwater (DW) isotopes at sites not shown in Figure $3.90 \%$ spread of simulations are from the 30 behavioural model runs. 

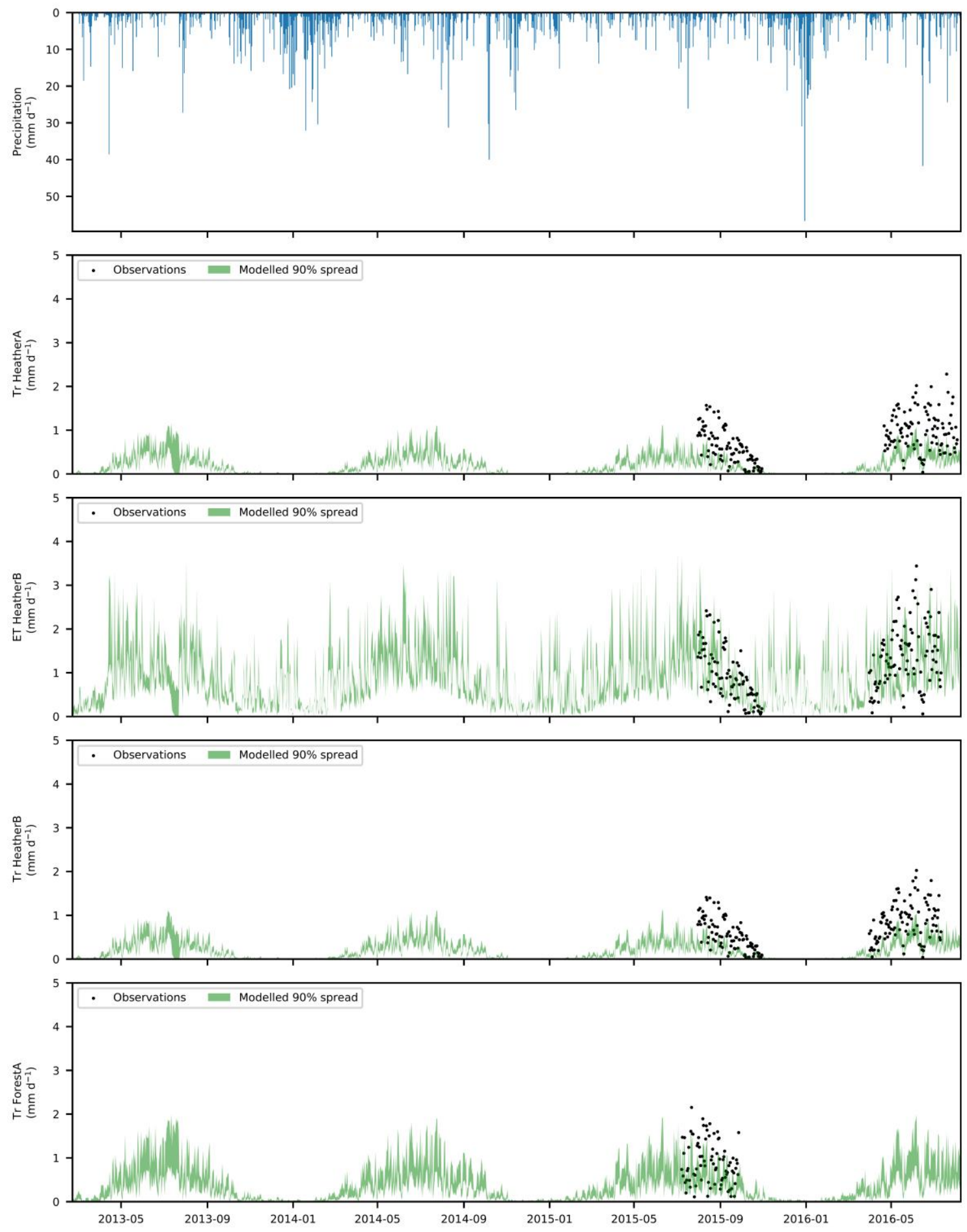

25 Figure S4: Time series of observed and simulated evapotranspiration (ET) and transpiration (Tr) at sites not shown in Figure 3. $90 \%$ spread of simulations are from the 30 behavioural model runs. 

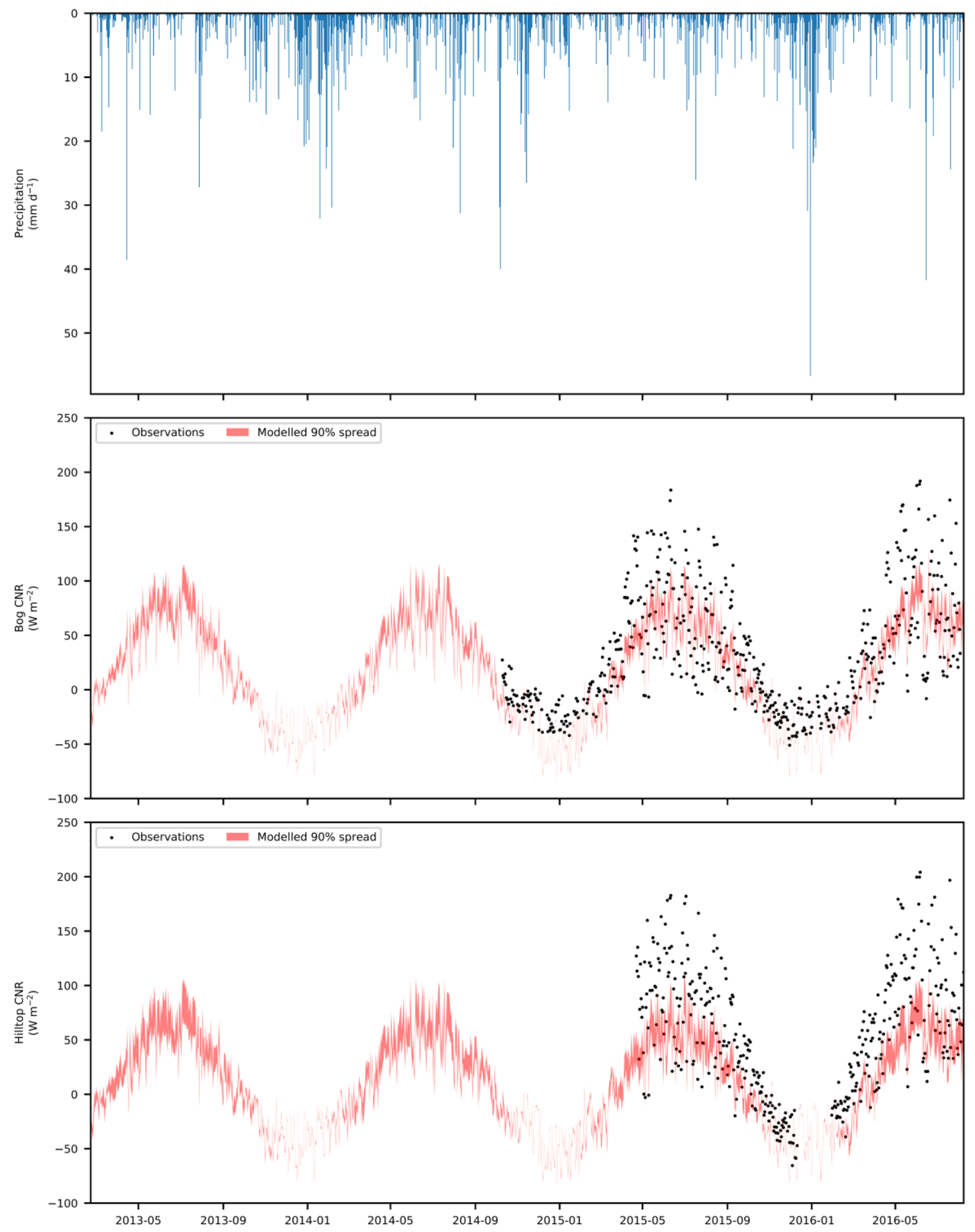

Figure S5: Time series of observed and simulated net radiation (CNR) at sites not shown in Figure 3. 90\% spread of simulations are from the 30 behavioural model runs. 
Capell, R., Tetzlaff, D., Soulsby, C., 2012. Can time domain and source area tracers reduce uncertainty in rainfall-runoff models in larger heterogeneous catchments? Water Resource Research 48, W09544. DOI: 10.1029/2011WR011543

Dee, D.P., Uppala, S.M., Simmons, A.J., Berrisford, P., Poli, P., Kobayashi, S., Andrae, U., Balmaseda, M.A., Balsamo, G., Bauer, P., Bechtold, P., Beljaars, A.C.M., van de Berg, L., Bidlot, J., Bormann, N., Delsol, C., Dragani, R., Fuentes, M., Geer, 35 A.J., Haimberger, L., Healy, S.B., Hersbach, H., Holm, E.V., Isaksen, L., Kållberg, P., Kohler, M., Matricardi, M., McNally, A.P., Monge-Sanz, B.M., Morcrette, J.-J., Park, B.-K., Peubey, C., de Rosnay, P., Tavolato, C., Thepaut, J.-N., Vitart, F., 2011. The ERA-Interim reanalysis: configuration and performance of the data assimilation system. Quarterly Journal of the Royal Meteorological Society 137: 553-597. DOI: 10.1002/qj.828

Met Office, 2017. Met Office Integrated Data Archive System (MIDAS) Land and Marine Surface Stations Data (185340 current). NCAS Br. Atmospheric Data Cent.

Summers, R.W., 2018. Abernethy Forest: The History and Ecology of an Old Scottish Pinewood. RSPB: Inverness.

Summers, R.W., Wilkinson, N.I., Wilson, E.R., 2008. Age structure and history of stand types of Pinus sylvestris in Abernethy Forest, Scotland. Scandinavian Journal of Forest Research 23: 28-37. DOI: 10.1080/02827580701646513 